\title{
Differences in displacement of the proximal and distal ends of mid-upper thoracic esophageal squamous cell carcinoma
}

\author{
GUOQIN QIU, DENGSHUN WEN, XIANGHUI DU, LIMING SHENG, XIA ZHOU, \\ YONGLING JI, WUAN BAO, DANHONG ZHANG and LEI CHENG
}

Department of Radiation Oncology, Zhejiang Cancer Hospital, Hangzhou, Zhejiang 310022, P.R. China

Received November 23, 2015; Accepted April 25, 2016

DOI: $10.3892 / \mathrm{mco} .2016 .899$

\begin{abstract}
In the present study, clips were used as markers to evaluate displacement differences between proximal and distal ends of esophageal tumors and to test whether their internal target volume (ITV) margins should be determined separately. A total of 23 patients with mid-upper thoracic esophageal squamous-cell carcinoma, a tumor length of $\leq 8 \mathrm{~cm}$ and an esophageal lumen suitable for endoscopic ultrasonography were recruited for the present study. Clips were implanted endoscopically at the proximal and distal ends of the esophageal tumor (upper and lower clips). In a further exploratory study on 16 of the patients, a third clip was placed at the distal esophagus $2 \mathrm{~cm}$ above the gastro-esophageal junction (GEJ) (cardiac clip). The clips were contoured for all 10 phases of the four-dimensional computed tomography and the maximum displacements of the clip centroids among different breathing phases in left-right (LR), superior-inferior (SI) and anterior-posterior (AP) directions were marked as $\mathrm{x}, \mathrm{y}$ and $\mathrm{z}$, respectively. The ITV margins that covered $95 \%$ of the LR, SI and AP motion were 2.89, 5.00 and $2.36 \mathrm{~mm}$, respectively. Axial displacement (y) was greater than radial displacement $(\mathrm{x}, \mathrm{z} ; \mathrm{P}<0.05)$. It was also revealed that $\mathrm{LR}(\mathrm{x}), \mathrm{SI}(\mathrm{y})$ and $\mathrm{AP}(\mathrm{z})$ displacement of cardiac clips was greater than that of upper or lower clips $(\mathrm{P}<0.05)$. Differences in the axial and radial displacement of the upper and lower clips indicated that axial and radial ITV margins should be determined separately. However, further study is required on patients in whom the distal tumor end is located in proximity to the GEJ.
\end{abstract}

\section{Introduction}

Esophageal cancer is one of the most common types of malignancy in China. Radiotherapy is commonly used concurrently with chemotherapy as either a definitive or pre-operative

Correspondence to: Dr Guoqin Qiu, Department of Radiation Oncology, Zhejiang Cancer Hospital, 38 Guangji Road, Hangzhou, Zhejiang 310022, P.R. China

E-mail: qiuguoqinaaa@126.com

Key words: esophageal cancer, four-dimensional computed tomography, clip, internal target volume treatment for esophageal carcinoma $(1,2)$. The International Commission on Radiation Units and Measurements report 62 defined the planning target volume and described two distinct components: i) The setup margin, which accounted for uncertainties in the patient position; and ii) the internal target volume (ITV), which accounted for variations in the size, shape and position of the tumor (3). Four-dimensional computed tomography (4D-CT) has improved the accuracy of measurements of the ITV for tumors that move during respiration, which allows for a more accurate adjustment of target volumes and a minimization of the radiation dose delivered to normal tissues, while avoiding a geographic miss $(4,5)$.

The motion of the centroid in all three dimensions has been quantitatively evaluated by 4D-CT scanning $(6,7)$. However, the esophagus is a long tubular organ extending from the neck to the upper abdomen, which is surrounded by a variety of structures. Thus, differences in displacement amplitudes of the esophagus across different segments are likely to be present (8-10). Esophageal tumors tend to grow and migrate longitudinally along the esophageal wall. Thus, we hypothesized that differential displacement of the esophageal tumor may also occur at its proximal and distal ends. For esophageal cancer, determination of tumor displacement at the proximal and distal end is particularly important, as it substantially represents tumor displacement as a whole. Thus, tumor centroid motion may not be representative of variations in the shape of the target or movement of its periphery and ITV margins in the context of the proximal and distal ends of the tumor, which should be determined separately. However, little is known regarding these variations in the setting of esophageal cancer.

Computed tomography has been shown to be relatively inaccurate in defining the proximal and distal ends of an esophageal lesion. Using a deformable image registration method, Zhao et al (6) evaluated respiratory-induced target motion of esophageal tumors at the gastro-esophageal junction (GEJ) and found that the deformed contours commonly featured several 'islands' located near the end when the reference shape of the gross tumor volume (GTV) was drawn flat with a large contour at the first or the final slice.

However, as deformable image registration methods were not available at all hospitals, previous studies have investigated the feasibility of using surgical clips to assess the volume and localization of the internal GTV based on 4D-CT images. A previous study has applied this approach for the assessment of 


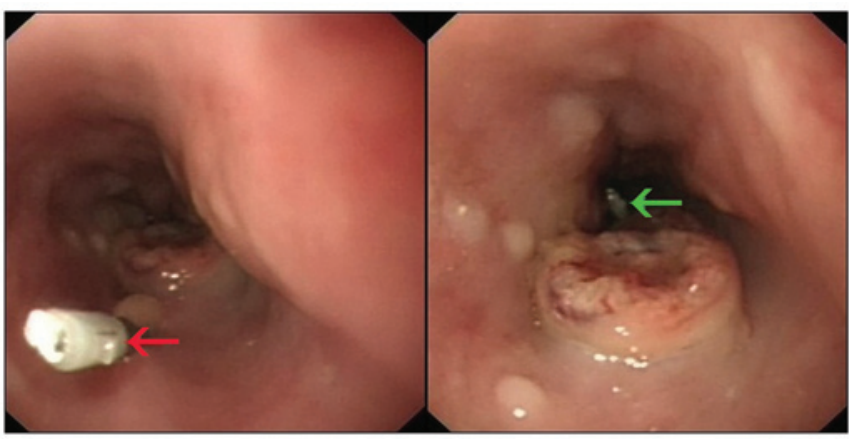

Figure 1. Endoscopically implanted upper clip (red arrow) and lower clip (green arrow).

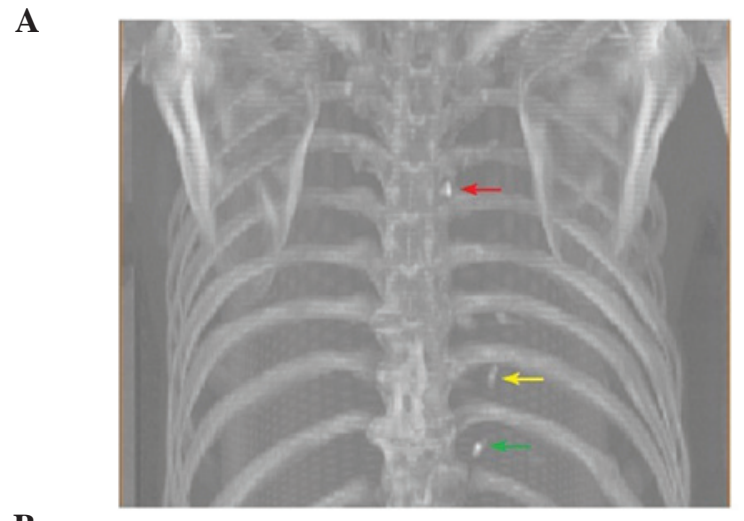

B

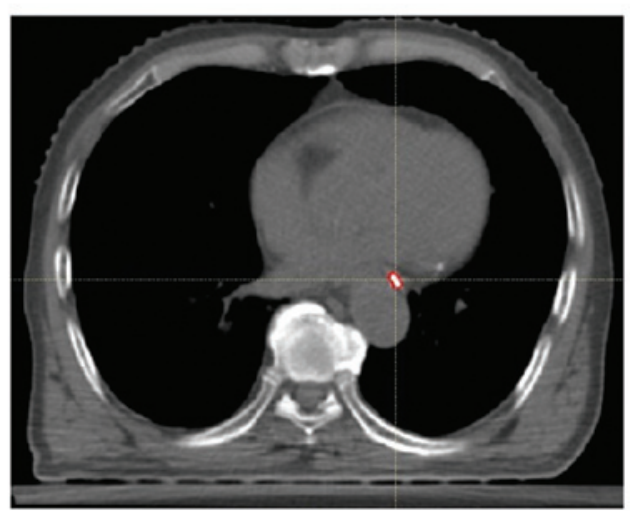

Figure 2. Outline and centroid of the clip. (A) Upper (red arrow), lower (yellow arrow) and cardiac (green arrow) clips as shown by digitally reconstructed radiography. (B) Outline (red) and centroid of a clip.

ITV margins with free breathing for external-beam partial breast irradiation in patients following breast-conserving surgery (11). Recently, bronchoscopy-assisted implantation of markers has been used for the determination of tumor-based setup during image-guided lung cancer radiotherapy with audiovisual biofeedback $(12,13)$. Metal objects are rigid, dense and well-defined, and provide a sharp contrast against the surrounding tissues, which they may be attached to, rendering them optimal and easily identifiable markers to track tumor motion.

A previous study revealed that a hemoclip technique may be safely used to accurately measure the longitudinal GTV of primary esophageal tumors (14).

The present study prospectively evaluated the displacement of endoscopically implanted clips at the proximal and distal ends of the tumor in patients with mid-upper thoracic
Table I. Clinical and tumor characteristics of the 23 patients.

\begin{tabular}{lc}
\hline Characteristics & $\mathrm{n}(\%)$ \\
\hline Age (years) & \\
Median & 64 \\
Gender & \\
Male & $21(91)$ \\
Female & $2(9)$ \\
Tumor stage & \\
T2 & $2(9)$ \\
T3 & $19(82)$ \\
T4 & $2(9)$ \\
Nodal stage & \\
N0 & $7(30)$ \\
N1 & $16(70)$ \\
Metastasis stage & \\
M0 & $15(65)$ \\
M1a & $8(35)$ \\
Lesion location & \\
Upper thoracic & $11(48)$ \\
Middle thoracic & $12(52)$ \\
Lesion length (cm) & \\
Mean \pm standard deviation & $3-8$ \\
Range & \\
\hline
\end{tabular}

esophageal squamous-cell carcinoma and compared differences in displacement between the upper and lower ends of the tumor in three dimensions. In this study, tumors located at the upper and middle thoracic esophagus were assessed, while it has been previously confirmed that tumors located in the distal esophagus are more mobile (9,10,15-17). Therefore, a further exploratory study was performed by placing a third clip at the lower thoracic esophagus at $2 \mathrm{~cm}$ above the non-neoplastic GEJ to preliminarily determine whether displacement differences between the upper and lower ends of the tumor may also occur if the distal end of the tumor is located near the GEJ.

\section{Patients and methods}

Study design and patients. A total of 23 patients with esophageal cancer who were treated at Zhejiang Cancer Hospital (Hangzhou, China) from September, 2012 to October, 2013 were recruited for this clinical study. The inclusion criteria were as follows: i) Diagnosed with mid-upper thoracic esophageal squamous-cell carcinoma with the upper edge of the tumor located at $<30 \mathrm{~cm}$ from the incisors; ii) T1-4, N0-1 and M0-1a stage according to the American Joint Committee on Cancer staging guidelines (18); iii) a lesion length of $\leq 8 \mathrm{~cm}$; iv) candidates for radical radiotherapy at the time of CT scanning; v) cases in which the endoscopic ultrasonography (EUS) probe was able to pass through the lumen of the esophageal lesions. Only a lesion length of $\leq 8 \mathrm{~cm}$ was considered as suitable for radical radiotherapy. Patients with multi-focal lesions were excluded from the study. Written informed consent was obtained from all subjects prior to treatment. 
Table II. Comparison of displacement of the upper, lower and cardiac clips.

\begin{tabular}{|c|c|c|c|c|c|c|c|}
\hline \multirow[b]{2}{*}{ Clips } & \multicolumn{2}{|c|}{$\mathrm{x}(\mathrm{mm})$} & \multicolumn{2}{|c|}{$\mathrm{y}(\mathrm{mm})$} & \multicolumn{2}{|c|}{$\mathrm{z}(\mathrm{mm})$} & \multirow[b]{2}{*}{ P-value } \\
\hline & Mean & $\mathrm{SD}$ & Mean & $\mathrm{SD}$ & Mean & SD & \\
\hline Upper & 1.04 & 0.72 & 2.54 & 1.66 & 0.80 & 0.59 & $<0.05$ \\
\hline Lower & 1.26 & 1.02 & 2.61 & 2.06 & 1.22 & 0.73 & $<0.05$ \\
\hline Cardiac & 2.27 & 1.06 & 5.23 & 2.43 & 2.07 & 1.09 & $<0.05$ \\
\hline P-value & \multicolumn{2}{|c|}{$<0.05$} & \multicolumn{2}{|c|}{$<0.05$} & \multicolumn{2}{|c|}{$<0.05$} & \\
\hline
\end{tabular}

$\mathrm{x}, \mathrm{y}$ and $\mathrm{z}$, maximum difference of clip centroid position among the 10 breathing phases in the left-right, superior-inferior and anterior-posterior directions, respectively; SD, standard deviation.

Clip implantation. Prior to 4D-CT simulator examination, the esophageal tumor was localized using an electronic gastroscope (GIF-Q260; Olympus, Tokyo, Japan). The clips were then positioned at the proximal and distal ends of the tumor and, in 16 of the patients, a third clip was placed at the lower thoracic esophagus $2 \mathrm{~cm}$ above the GEJ (Fig. 1).

4D-CT simulation and image acquisition. The patients were placed in the supine position with their arms placed at their sides, with a standard wing board that was used for immobilization. The Varian Real-time Position Management (RPM) system (Varian Medical Systems, Palo Alto, CA, USA) was used to monitor the breathing cycle. All CT images were obtained from the middle neck to the upper abdomen at a 3-mm slice thickness upon completion of the standard CT simulation using a 16-slice Brilliance big bore CT scanner (Philips Medical Systems, Inc., Cleveland, OH, USA) during normal breathing in a resting state. Following image acquisition, the $\mathrm{CT}$ images were reconstructed for the corresponding respiratory phases according to the respiratory signal externally acquired by RPM and all CT images were then automatically sorted into 10 categories that corresponded to the respiratory phase at which the image was captured using Varian 4D Integrated Treatment Console Version 13.0 (GE Healthcare, Little Chalfont, UK).

Clip contouring. Upper, lower and cardiac clips were contoured using a mediastinal window setting for each patient and for all 10 breathing phases of each 4D-CT on a Raystation treatment-planning workstation (version 4.5.1; Raysearch Laboratories AB, Stockholm, Sweden). Delineation of clips was performed semi-automatically (using smart contour software) and then manually edited by a single radiation oncologist (Fig. 2).

Measurement of clip displacement. To quantify the motion of the clips, the centroid of each clip was calculated using commercial treatment planning system software (Raystation version 4.5.1). The centroids of all three markers were recorded at the 10 respiratory phases. The maximum differences of the clip centroid position among the 10 breathing phases in the left-right (LR), superior-inferior (SI) and anterior-posterior (AP) directions were determined and referred to as $\mathrm{x}, \mathrm{y}$ and $\mathrm{z}$, respectively.
Margin expansions for ITV coverage. The minimum expansion necessary to cover the ITVs of $\sim 95 \%$ of the tumors in the LR, SI and AP dimensions was calculated.

Statistical analysis. Values are expressed as the mean \pm standard deviation. SPSS version 17.0 software (SPSS, Inc., Chicago, IL, USA) was used for statistical analyses. For each clip, the displacement of the clip centroid in three dimensions ( $x, y$ and z) was statistically compared by least-significant differences one-way analysis of variance. The latter method was also used to compare the displacements among the upper, lower and cardiac clips in one direction. $\mathrm{P}<0.05$ was considered to indicate a statistically significant difference.

\section{Results}

Patient characteristics. The characteristics of the patients are listed in Table I. All the patients had upper and lower clips, and 16 of the patients also had cardiac clips. None of the patients presented with esophageal bleeding or perforation. During the CT-based simulation, all clips remained attached.

Comparison of displacements in three directions for each clip. For the upper clips, the displacement in LR, SI and AP direction was $1.04 \pm 0.72,2.54 \pm 1.66$ and $0.80 \pm 0.59 \mathrm{~mm}$, respectively. For the lower clips, the displacement in LR, SI and AP direction was $1.26 \pm 1.02,2.61 \pm 2.06$ and $1.22 \pm 0.73 \mathrm{~mm}$, respectively. For the cardiac clips, the displacement in LR, SI and AP direction was $2.27 \pm 1.06,5.23 \pm 2.43$ and $2.07 \pm 1.09 \mathrm{~mm}$, respectively. For each clip, the axial displacement (y) was significantly greater than the radial displacement $(\mathrm{x}, \mathrm{z} ; \mathrm{P}<0.05)$ and there were no significant differences between the radial displacements ( $\mathrm{x}, \mathrm{z}$; $\mathrm{P}>0.05$ ) (Table II).

Comparison of displacements among the three clips. No significant differences in displacement were found between the upper and lower clips in $\mathrm{x}, \mathrm{y}$ and $\mathrm{z}$ direction. However, cardiac clips differences in displacement in $\mathrm{x}, \mathrm{y}$ and $\mathrm{z}$ direction were greater than those of the upper or lower clips $(\mathrm{P}<0.05)$ (Table II).

Margin expansions for ITV coverage. The minimum expansion required to cover the ITVs of $95 \%$ of the tumors in the LR, SI and AP directions was 2.89, 5.00 and $2.36 \mathrm{~mm}$, respectively. 


\section{Discussion}

In the present study, clips were used as markers to define the ITV margins of the proximal and distal ends of esophageal tumors that occurred at the mid-upper thoracic esophagus. The results revealed that the axial displacement was significantly greater than the radial displacement and asymmetric margins to cover $\sim 95 \%$ of RL, SI and AP motion were 2.89, 5.00 and $2.36 \mathrm{~mm}$, respectively. The proximal and distal ends of the tumors had a similar magnitude of displacement in the RL, SI and AP direction. Thus, it is not recommended to set differential ITV margins based on different parts of the tumor for mid-upper esophageal cancer.

Several studies have previously reported directional displacement differences in primary esophageal cancer $(6,10,15)$. Zhao et al (6) studied tumors located at the GEJ using respiratory-induced target motions by 4D-CT and recommended margins of $1.0 \mathrm{~cm}$ (left), $0.8 \mathrm{~cm}$ (right), $1.1 \mathrm{~cm}$ (anterior), $0.6 \mathrm{~cm}$ (posterior), $1.0 \mathrm{~cm}$ (superior) and $1.6 \mathrm{~cm}$ (inferior). Hashimoto et al (10) also found that the mean range of motion of fiducial markers inserted into the esophageal wall was $3.5 \pm 1.8 \mathrm{~mm}, 8.3 \pm 3.8$ and $4.0 \pm 2.6 \mathrm{~mm}$, respectively, for the medio-lateral, cranio-caudal and AP directions. Furthermore, a study by Patel et al (15) using time-resolved 4D-CT revealed that the peak-to-peak displacements of all primary tumors in the SI, AP and LR dimensions were $0.80 \pm 0.45,0.28 \pm 0.20$ and $0.22 \pm 0.23 \mathrm{~cm}$, respectively. The results of the present study were concordant with those determined in the abovementioned studies, while the magnitude of motion in the SI direction $(5.00 \mathrm{~mm})$ determined by the present study was smaller. This was partly due to the fact that the lesions of all patients of the present study were located at the mid-upper thoracic esophagus, which have been reported to have a reduced motion compared to those at the distal esophagus $(9,10)$.

To the best of our knowledge, the present study was the first to evaluate whether displacement differences exist between the upper and lower ends of an esophageal tumor that is located at the upper-middle thoracic esophagus. Patients with this type of esophageal cancer are considered to be prime candidates for definitive radiotherapy, highlighting the importance of the findings of the present study. Our results demonstrated that no significant differences in displacement were found between the upper and lower clips in the LR, SI and AP directions and, thus, differential ITV margins based on different parts of the tumor are not recommended. Previous studies have investigated the displacement of tumors in various parts of the esophagus with conflicting results $(9,10,15-17)$. For example, in a study by Lorchel et al (16), 8 patients that presented with esophageal malignancies underwent two conventional spiral CT scans during breath-hold procedure under spirometric control, and no association of tumor motion with anatomical location was observed.

Other studies have reported that tumors in the distal esophagus had higher mobility compared with those in the middle and upper esophagus $(9,10,15,17)$. The aim of the present study was to investigate the displacement differences between the superior and inferior ends of tumors located in the upper-middle thoracic esophagus in three directions. However, the results were not in agreement with two previous studies reporting that intrafractional esophageal displacement varied depending on the location in the normal esophagus $(8,9)$. This finding allows for the hypothesis that the neoplastic esophagus moves in different ways from the healthy esophagus.

However, further analysis using clips at the lower thoracic esophagus near the GEJ revealed a significantly larger motion in three directions. This suggested that the ITV margins of the superior and inferior ends of esophageal tumors should be determined separately in patients in wom the lower edge of the lesion is located near the GEJ. A study by Hashimoto et al (10) evaluating the feasibility of real-time monitoring of fiducial markers in the digestive tract followed by analysis of the motion of the healthy esophagus concluded that respiration and heartbeat (particularly) were the main causes of esophageal motion.

Furthermore, the esophagus near the GEJ may be subject to considerable respiratory diaphragmatic motion, becoming more mobile. However, the observations of the present study should be interpreted with caution, as the data of this exploratory study were derived from the displacement of clips attached to the normal distal esophagus, which may be mobile in ways that are different from the esophagus bearing tumors. Therefore, a further prospective study is required to assess the displacement of clips attached to tumors whose distal end is located in proximity to the GEJ. Furthermore, the accuracy of the calculation of the marker centroids was limited, considering that the use of 3-mm CT slices affected the accuracy of the position of the markers by $1.0 \mathrm{~cm}$. These errors may be reduced by acquiring $\mathrm{CT}$ images with a lower axial slice thickness or by volumetric acquisition $(17,19)$.

In conclusion, to the best of our knowledge, the present study was the first to use clips and 4D-CT imaging to determine the ITV margins of upper-middle esophageal cancer. The minimum expansion of ITV margins required to cover $95 \%$ of the RL, SI and AP motion were calculated to be 2.89, 5.00 and $2.36 \mathrm{~mm}$, respectively. For the upper and lower clips, axial displacement (y) was greater than radial displacement $(\mathrm{x}, \mathrm{z} ; \mathrm{P}<0.05)$, indicating that axial and radial ITV margins should be determined separately. It was also revealed that LR, SI and AP displacement of clips attached to the normal GEJ was greater than that of upper or lower clips $(\mathrm{P}<0.05)$; therefore, further study is required on patients in whom the distal tumor end is located in proximity to the GEJ.

\section{References}

1. Minsky BD, Pajak TF, Ginsberg RJ, et al: INT 0123 (Radiation Therapy Oncology Group 94-05) phase III trial of combined-modality therapy for esophageal cancer: High-dose versus standard-dose radiation therapy. J Clin Oncol 20: 1167-1174, 2002.

2. van Hagen P, Hulshof MC, van Lanschot JJ, et al: Preoperative chemoradiotherapy for esophageal or junctional cancer. N Engl J Med 366: 2074-2084, 2012.

3. International Commission on Radiation Units and Measurements. ICRU report 62: Prescribing, recording and reporting photon beam therapy (supplement to ICRU report 50). Washington, DC: ICRU, 1999.

4. Admiraal MA, Schuring D and Hurkmans CW: Dose calculations accounting for breathing motion in stereotactic lung radiotherapy based on 4D-CT and the internal target volume. Radiother Oncol 86: 55-60, 2008

5. Yakoumakis N, Winey B, Killoran J, Mayo C, Niedermayr T, Panayiotakis G, Lingos T and Court L: Using four-dimensional computed tomography images to optimize the internal target volume when using volume-modulated arc therapy to treat moving targets. J Appl Clin Med Phys 13: 3850, 2012. 
6. Zhao KL, Liao Z, Bucci MK, Komaki R, Cox JD, Yu ZH, Zhang L, Mohan R and Dong L: Evaluation of respiratory-induced target motion for esophageal tumors at the gastroesophageal junction. Radiother Oncol 84: 283-289, 2007.

7. Yaremko BP, Guerrero TM, McAleer MF, Bucci MK, Noyola-Martinez J, Nguyen LT, Balter PA, Guerra R, Komaki R and Liao Z: Determination of respiratory motion for distal esophagus cancer using four-dimensional computed tomography. Int J Radiat Oncol Biol Phys 70: 145-153, 2008.

8. Pan CC, Kashani R, Hayman JA, et al: Intra- and inter-fraction esophagus motion in 3D-conformal radiotherapy: Implications for ICRU 62 definitions of internal target volume and planning organ at risk volume. Int J Radiat Oncol Biol Phys 60 (Suppl): S580-S581, 2004.

9. Dieleman EM, Senan S, Vincent A, Lagerwaard FJ, Slotman BJ and van Sörnsen de Koste JR: Four-dimensional computed tomographic analysis of esophageal mobility during normal respiration. Int J Radiat Oncol Biol Phys 67: 775-780, 2007.

10. Hashimoto T, Shirato H, Kato M, Yamazaki K, Kurauchi N, Morikawa T, Shimizu S, Ahn YC, Akine Y and Miyasaka K: Real-time monitoring of a digestive tract marker to reduce adverse effects of moving organs at risk (OAR) in radiotherapy for thoracic and abdominal tumors. Int J Radiat Oncol Biol Phys 61: 1559-1564, 2005.

11. Ding Y, Li J, Wang W, Wang S, Wang J, Ma Z, Shao Q and Xu M A comparative study on the volume and localization of the internal gross target volume defined using the seroma and surgical clips based on 4DCT scan for external-beam partial breast irradiation after breast conserving surgery. Radiat Oncol 9: 76, 2014.

12. Roman NO, Shepherd W, Mukhopadhyay N, Hugo GD and Weiss E: Interfractional positional variability of fiducial markers and primary tumors in locally advanced non-small-cell lung cancer during audiovisual biofeedback radiotherapy. Int J Radiat Oncol Biol Phys 83: 1566-1572, 2012.

13. Ueki N, Matsuo Y, Nakamura M, Mukumoto N, Iizuka Y, Miyabe Y, Sawada A, Mizowaki T, Kokubo M and Hiraoka M: Intra- and interfractional variations in geometric arrangement between lung tumours and implanted markers. Radiother Oncol 110: 523-528, 2014.
14. Qiu GQ, Du XH, Yu JP, Zheng YD, Luo HJ, Xu YP, Chen JX, Sun XJ, Ji YL and Tao YL: The value of endoscopic ultrasonography in defining longitudinal gross target volumes for esophageal squamous carcinoma. Surg Laparosc Endosc Percutan Tech 22: 424-428, 2012.

15. Patel AA, Wolfgang JA, Niemierko A, Hong TS, Yock T and Choi NC: Implications of respiratory motion as measured by four-dimensional computed tomography for radiation treatment planning of esophageal cancer. Int J Radiat Oncol Biol Phys 74: 290-296, 2009.

16. Lorchel F, Dumas JL, Noël A, Wolf D, Bosset JF and Aletti P: Esophageal cancer: Determination of internal target volume for conformal radiotherapy. Radiother Oncol 80: 327-332, 2006.

17. Yamashita H, Kida S, Sakumi A, Haga A, Ito S, Onoe T, Okuma K, Ino K, Akahane M, Ohtomo K and Nakagawa K: Four-dimensional measurement of the displacement of internal fiducial markers during 320-multislice computed tomography scanning of thoracic esophageal cancer. Int J Radiat Oncol Biol Phys 79: 588-595, 2011.

18. Greene FL, Page DL, Fleming ID, et al: AJCC Cancer Staging Manual. 6th edition. Springer-Verlag, New York, 2002.

19. Yamashita H, Okuma K, Tada K, Shiraishi K, Takahashi W, Shibata-Mobayashi S, Sakumi A, Saotome N, Haga A, Onoe $\mathrm{T}$, et al: Four-dimensional measurement of the displacement of internal fiducial and skin markers during 320-multislice computed tomography scanning of breast cancer. Int J Radiat Oncol Biol Phys 84: 331-335, 2012. 